\title{
Effect of the nuclear hyperfine field on the 2D electron conductivity in the quantum Hall regime
}

\author{
S. A. Vitkalov \\ Chemistry Department and National High Magnetic Field Laboratory, University of \\ Florida, Gainesville, Florida 32611-7200 USA; \\ P. N. Lebedev Physical Institute, Russian Academy of Sciences, 117924 Moscow, Russia \\ C. R. Bowers \\ Chemistry Department and National High Magnetic Field Laboratory, University of \\ Florida, Gainesville, Florida 32611-7200 USA
}

\author{
J. A. Simmons and J. L. Reno \\ Sandia National Laboratories, Albuquerque, NM 87185-1415
}

(Submitted 19 November 1998; resubmitted 2 December 1998)

Pis'ma Zh. Éksp. Teor. Fiz. 69, No. 1, 58-63 (10 January 1999)

The effect of the nuclear hyperfine interaction on the dc conductivity of 2D electrons under quantum Hall effect conditions at filling factor $\nu=1$ is observed for the first time. The local hyperfine field enhanced by dynamic nuclear polarization is monitored via the Overhauser shift of the 2D conduction electron spin resonance in AlGaAs/GaAs multiquantum-well samples. The experimentally observed change in the dc conductivity resulting from dynamic nuclear polarization is in agreement with a thermal activation model incorporating the Zeeman energy change due to the hyperfine interaction. The relaxation decay time of the dc conductivity is, within experimental error, the same as the relaxation time of the nuclear spin polarization determined from the Overhauser shift. These findings unequivocally establish the nuclear spin origins of the observed conductivity change. (c) 1999 American , Institute of Physics. [S0021-3640(99)01201-3]

PACS numbers: $73.40 . \mathrm{Hm}, 72.15 .-\mathrm{v}, 73.20 . \mathrm{Dx}$

In conductors and superconductors, fluctuations of the hyperfine contact interaction usually provide the dominant mechanism for nuclear spin relaxation at low temperatures. ${ }^{1,2}$ These relaxation times are closely related to the electronic structure and dynamics in these materials. In recent years it has been recognized that the hyperfine interaction can serve as a powerful tool in studies of $2 \mathrm{D}$ conducting electron systems. Several notable applications include the quantum Hall effect (QHE) ${ }^{3}$ and quantum computation. ${ }^{4}$ However, studies of the effect of electron-nuclear spin interactions on the electronic transport are very rare. In nonmagnetic metals the spin-spin interaction between electrons and nuclei is theoretically predicted to produce a strongly magnetic-field and temperature dependent contribution to the resistivity. ${ }^{5}$ The contribution of the hyperfine interaction to magnetotransport quantum oscillations of the resistivity has been ob- 


\section{DISCLAIMER}

This report was prepared as an account of work sponsored by an agency of the United States Government. Neither the United States Government nor any agency thereof, nor any of their employees, make any warranty, express or implied, or assumes any legal liability or responsibility for the accuracy, completeness, or usefulness of any information, apparatus, product, or process disclosed, or represents that its use would not infringe privately owned rights. Reference herein to any specific commercial product, process, or service by trade name, trademark, manufacturer, or otherwise does not necessarily constitute or imply its endorsement, recommendation, or favoring by the United States Government or any agency thereof. The views and opinions of authors expressed herein do not necessarily state or reflect those of the United States Government or any agency thereof. 


\section{DISCLAIMER}

Portions of this document may be illegible in electronic image products. Images are produced from the best available original document. 
served in bulk InSb. ${ }^{67}$ In a $2 \mathrm{D}$ electron system the effect of hyperfine interaction on electron spin resonance (Overhauser effect) has been observed in AlGaAs/GaAs. ${ }^{8,9}$ In the present study we demonstrate experimentally that the hyperfine interaction can produce an observable change of the dc conductivity of a $2 \mathrm{D}$ electron system under quantum Hall conditions.

To predict the effect of the hyperfine field on the $2 \mathrm{D}$ conductivity we use the conventional assumption that the energy of the excitations of the ground state in the QHE near filling factor $\nu=1$ can be expressed as a sum of two terms: $:^{10,11}$

$$
\Delta=\Delta_{0}+|g| \mu_{B}\left(B_{0}+B_{n}\right),
$$

where $\Delta_{0}$ is the exchange energy due to $e-e$ interaction and $|g| \mu_{B} B_{0}$ is the Zeeman term due to the externally applied field $B_{0}$. Through the hyperfine field the nuclei provide an additional contribution $|g| \mu_{B} B_{n}$ to the $2 \mathrm{D}$ ground state excitation energy. Here $g$ is the Landé $g$ factor of the excitations and $\mu_{B}$ is the electron Bohr magneton. The local hyperfine field $B_{n}$ is proportional to the nuclear spin polarization, $B_{n}=a\left\langle I_{z}\right\rangle,{ }^{12,13}$ where $a$ is the contact hyperfine coupling constant. At temperatures much greater than a few $\mathrm{mK}$, the thermal equilibrium hyperfine field $B_{n}^{\text {eq }}$ can ordinarily be neglected. In the context of electron spin resonance (ESR), $B_{n}$ is known as the Overhauser shift. ${ }^{8,13.14}$ In the absence of spin-orbit interaction, as in the conduction band of GaAs, ${ }^{11,15}$ neither the cyclotron energy nor the electron-electron Coulomb interactions are affected by $B_{n}$, regardless of its magnitude or sign, because the origin of $B_{n}$ is the spin-spin coupling between the electron and nucleus.

Under our experimental conditions, where $T \approx 2.5 \mathrm{~K}$ and $B_{0}=5.35 \mathrm{~T}$, the longitudinal conductivity at $\nu=1$ obeys an Arrhenius law:

$$
\sigma_{x x}=\sigma_{0} \exp (-\Delta / 2 k T) \text {, }
$$

where $\sigma_{0}$ is a constant. In the thermally activated regime the energy gap $\Delta$ can be determined from the temperature dependence of $\sigma_{x x}$. Consider the conductivity change that would result from a change in the nuclear polarization: through the collective hyperfine interaction of the 2D electrons with the nuclei in the vicinity of the 2D electrons, the local nuclear hyperfine field $B_{n}$ will be enhanced. For a small change in the hyperfine field, $\delta B_{n} \ll B_{0}$, we will have from (1) and (2):

$$
\delta \sigma_{x x} / \sigma_{x x}=-|g| \mu_{B} \delta B_{n} / 2 k T=\alpha \delta B_{n} / B_{0},
$$

where $\alpha=-|g| \mu_{B} B_{0} / 2 k T$ is a constant. Experimentally we measured the dc conductivity of the AlGaAs/GaAs multiquantum well samples by the standard four probe method in magnetic fields up to $B_{0}=6 \mathrm{~T}$ and temperatures $T=1.7-4.2 \mathrm{~K}$. To obtain the 2D longitudinal conductivity $\sigma_{x x}$ we measured the longitudinal resistivity $\rho_{x x}=\beta U_{x x} / I$ and the Hall resistivity $\rho_{x y}=U_{x y} / I$, where $\beta$ is a geometric factor, $I$ is the current through the sample, and $U_{x x}$ and $U_{x y}$ are the longitudinal and Hall voltages. To calculate the $2 \mathrm{D}$ conductivity we used the standard formula $\sigma_{x x}=\rho_{x x} /\left(\rho_{x x}^{2}+\rho_{x y}^{2}\right)$.

According to (3) the relative dc conductivity change is proportional to the change in the nuclear hyperfine field $B_{n}$. To observe this dependence experimentally, we have employed the method of dynamic nuclear polarization (DNP) by down-field swept ESR to enhance the magnitude of $\left\langle I_{z}\right\rangle^{8,9}$ The corresponding DNP-enhanced hyperfine field is $B_{n}^{\mathrm{DNP}}$. The change in the Overhauser shift of the ESR line is given by $\delta B_{n}=B_{n}^{\mathrm{DNP}}$ 

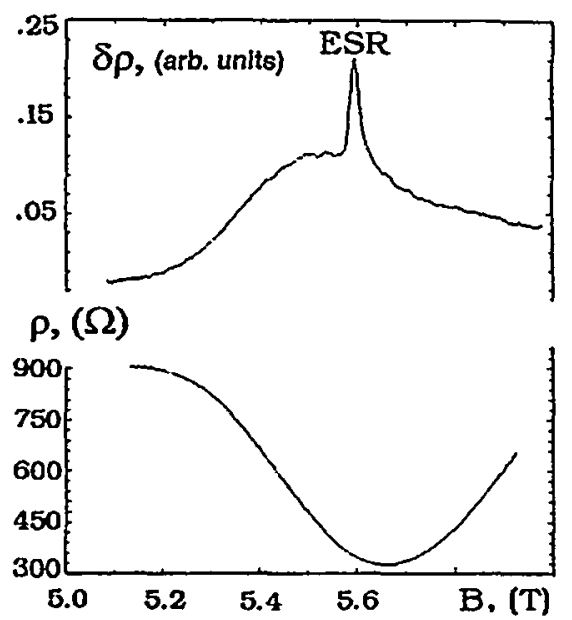

FIG. 1. Magnetic field dependence of the longitudinal resistivity per layer (lower curve) and variation of the resistivity induced by microwave excitation near filling factor $\nu=1$ in sample EA124. The ESR line position corresponds to $g=-0.415$. The spectrum was recorded at a microwave frequency of $32.48 \mathrm{GHz}$ and at $T=1.7 \mathrm{~K}$. The angle between $B_{0}$ and the normal to the sample is $60^{\circ}$.

$-B_{n}^{\text {eq }}$ (Refs. 8, 13, and 14). The 2D electron spin resonance signal is detected electrically as described in a previous paper ${ }^{16}$ on single $\mathrm{AlGaAs} / \mathrm{GaAs}$ heterojunctions. The mechanism for this phenomenon is not yet fully understood, but it nevertheless provides a working method to detect and control the Zeeman energy term. ${ }^{8,9}$

We have studied two different GaAs/AlGaAs multiquantum well samples. The samples were grown by molecular beam epitaxy and have the following properties at $T=4.2 \mathrm{~K}$ : sample EA124 (21 GaAs wells) has a $2 \mathrm{D}$ electron density per layer of $6.9 \times 10^{10} \mathrm{~cm}^{-2}$ and mobility $\mu=440000 \mathrm{~V} / \mathrm{cm}^{2}$ s; sample EA216 (40 GaAs wells) has a 2D density of $1.2 \times 10^{11} \mathrm{~cm}^{-2}$ and mobility $\mu=650000 \mathrm{~V} / \mathrm{cm}^{2} \mathrm{~s}$. The samples were mounted on a rotation stage to allow $\nu=1$ to be obtained over a range of magnetic fields. We measured $\rho_{x x}$ at a frequency of $524 \mathrm{~Hz}$ using a lock-in amplifier. Application of the microwave power to the sample induced a change in $\rho_{x x}$ which is detected on the output signal of the amplifier. To increase the sensitivity for ESR detection, the microwave power was modulated at a frequency of $7 \mathrm{~Hz}$. The output of this amplifier was connected to the input of a second lock-in amplifier which detected $\Delta \rho_{x x}$ induced by the microwave excitation at $7 \mathrm{~Hz}$. This method helps to avoid the rectification signal at $7 \mathrm{~Hz}$ which is induced by the microwave electromagnetic field. In Fig. 1 we present an example of the electrically detected $2 \mathrm{D}$ electron spin resonance at filling factor $\nu=1$, recorded using a microwave frequency of $32.48 \mathrm{GHz}$. Also shown is the longitudinal magnetoresistance at $T=1.7 \mathrm{~K}$ for sample EA124.

To polarize the nuclei the microwave generator was switched to continuous mode with no modulation. ${ }^{16}$ The magnetic field was first set to a value slightly higher than the ESR condition, and the field was then swept down. The resonant microwaves begin to polarize the nuclei through saturation of ESR transitions. ${ }^{8,13,14}$ As the nuclear polarization 

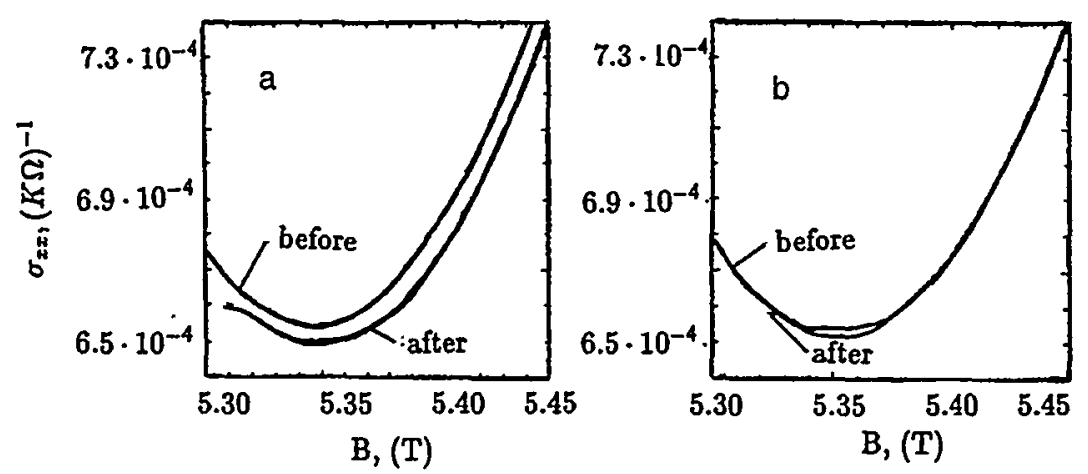

FIG. 2. Magnetic field dependences of the 2D longitudinal conductivity per layer before and after microwave irradiation of the sample, obtained at $T=2.5 \mathrm{~K}$ in sample EAI24. In experiment (a) the nuclei were dynamically polarized by resonant saturation of the electron spin transition. A microwave frequency of $32 \mathrm{GHz}$ was employed, corresponding to ESR at $5.5 \mathrm{~T}$ with the nuclei at thermal equilibrium. In experiment (b), the conductivity was recorded before and after off-resonant microwave excitation of the sample. The same microwave power was applied as in part (a), but the microwave frequency of $20.8 \mathrm{GHz}$ (corresponding to an ESR field of $3.58 \mathrm{~T}$ ) was far from the ESR condition, resulting in no dynamic nuclear polarization enhancement.

increases, the hyperfine field $B_{n}$ due to the nuclei acting on the electrons also increases, resulting in the Overhauser shift of the ESR line. In GaAs this shift is to lower magnetic field, ${ }^{8,17}$ because $B_{n}$ is positive and adds constructively to $B_{0}$ due to the negative sign of the $g$ factor. By choosing an appropriate down-sweep rate, it is possible to shift the ESR line considerably. ${ }^{8}$ The maximum ESR shift that we obtained by microwave DNP was $B_{n}=0.2 \mathrm{~T}$ at $B_{0}=5.5 \mathrm{~T}$.

In accordance with (3) the enhancement of the nuclear hyperfine field $B_{n}$ should change the longitudinal dc conductivity $\sigma_{x x}$ of the $2 \mathrm{D}$ electrons under $\mathrm{QHE}$ conditions. In Fig. 2 we present the magnetic field dependences of $\sigma_{x x}$ for EA124 at $T=2.5 \mathrm{~K}$. To observe the change $\delta \sigma_{x x}$ due to $\delta B_{n}$, we first measured $\sigma_{x x}^{\text {eq }}\left(B_{0}\right)$ with a magnetic field up-sweep without microwave excitation, with the nuclei in thermal equilibrium with the lattice. This conductivity trace is labeled before in Fig. 2a. To ensure that the nuclear spin system was initially at thermal equilibrium with the lattice, the $2 \mathrm{D}$ electron system was taken just outside of the $\nu=1$ magnetoresistance minimum for 300-600 $\mathrm{s}$ before the first sweep. The relaxation of the Overhauser shift of the ESR indicates that the nuclear spin relaxation time $T_{1 n}$ is about $60-120 \mathrm{~s}$ under these conditions, in reasonable agreement with previous reports. ${ }^{9}$ After $\sigma_{x x}^{\text {eq }}\left(B_{0}\right)$ was recorded in the first sweep, the nuclei were dynamically polarized by the down-swept DNP procedure ${ }^{8}$ at a sweep rate $d B / d t=0.5$ $\mathrm{mT} / \mathrm{s}$ near $\nu=1$. The microwave power was then switched off, and $\sigma_{x x}^{\mathrm{DNP}}\left(B_{0}\right)$ was recorded during a second magnetic field up-sweep, this time with the nuclear spin system highly polarized (Fig. 2a). This is the trace labeled after in Fig. 2a.

Using electrically detected ESR we also measured the Overhauser shift of the ESR line as a function of time using multiple detection up-sweeps. The relaxation time of the Overhauser shift at $\nu=1$ and $T=2.7 \mathrm{~K}$ was observed to be $240-300 \mathrm{~s}$. Since this is much longer than the time scale of the ESR detection up-sweep (about $30 \mathrm{~s}$ ), the amount of 
nuclear spin relaxation which occurs during the up-sweep is small and can be neglected for the purposes of this paper.

To eliminate the possibility that the observed time evolution of the conductivity is due to some sort of persistent microwave photoconductivity effect, the dc conductivity was measured using exactly the same procedure as in the resonant DNP experiment but with nonresonant microwave excitation of the sample at frequencies far from the ESR condition. The result is that nonresonant microwave excitation changes neither position of the ESR line nor the dc conductivity of sample EA124. The conductivity traces obtained before and after nonresonant excitation are therefore overlapping, as is evident from Fig. $2 b$.

We now estimate the value of the relative dc conductivity change due to the change $\delta B_{n}$ induced by DNP according to (3) and compare this with the data represented in Fig. 2a. Let $B_{0}^{S}$ be the magnetic field satisfying the ESR condition in the absence of the hyperfine field (e.g., nuclei unpolarized). The difference between the ESR fields before (at thermal equilibrium) and immediately after enhancement of the nuclear polarization is given by $\delta B_{n}=\left(B_{0}^{s}-B_{n}^{\mathrm{eq}}\right)-\left(B_{0}^{s}-B_{n}^{\mathrm{DNP}}\right)=B_{n}^{\mathrm{DNP}}-B_{n}^{\mathrm{eq}}$. The observed time dependence of the Overhauser shift demonstrates that the position of the ESR line immediately after switching off the microwaves at the end of the DNP down-sweep coincides well with $B_{0}^{s}-B_{n}^{\mathrm{DNP}}$. In the experiment corresponding to Fig. 2a, the ESR was shifted from its equilibrium position $B_{0}^{s}-B_{n}^{\mathrm{eq}}=5.503 \mathrm{~T}$ to $B_{0}^{s}-B_{n}^{\mathrm{DNP}}=5.304 \mathrm{~T}$, and therefore $\delta B_{n}=199 \mathrm{mT}$. In accordance with relation (3) the dc conductivity variation due to $B_{n}^{\text {DNP }}$ should be $\delta \sigma_{x x}^{\text {theor }}=7.3 \times 10^{-6} \mathrm{k} \Omega^{-1}$. The $g$ factor $g=-0.415$ used to calculate $\delta \sigma_{x x}^{\text {theor }}$ was determined from ESR (see Fig. 1). Using the experimental data from Fig. 2a, the dc conductivity change due to $\delta B_{n}$ was found to be $\delta \sigma_{x x}^{\exp }=8 \times 10^{-6} \mathrm{k} \Omega^{-1}$. This value corresponds to the difference between the minima of the $\sigma_{x x}\left(B_{0}\right)$ curves before and after enhancement of the nuclear spin polarization. Hence, there is reasonable agreement between the experiment and the simple theoretical estimate of the conductivity change due to DNP enhancement of the nuclear polarization.

Figure 3a presents the relaxation decay of the change in the ESR position $\delta B_{n}(t) / B_{0}^{s}$ for sample EA216, obtained from ESR by multiple magnetic field up-sweeps during a period of $800 \mathrm{~s}$ immediately following the initial DNP down-sweep. The time dependence was fit to an exponential decay function: $\delta B_{n}(t) \propto \exp \left(-t / T_{1 n}\right)$, yielding $T_{1 n}$ $=217 \mathrm{~s}$ at $\nu=1$ and $T=2.5 \mathrm{~K}$. The time $T_{1 n}$ is comparable with the relaxation times obtained in Ref. 9.

The relaxation decay of the dc conductivity change of EA216 immediately following the DNP down-sweep is presented in Fig. 3b. The relative variation of the conductivity $\delta \sigma_{x x} / \sigma_{x x}=\left(\sigma_{x x}(t)-\sigma_{x x}^{\mathrm{eq}}\right) / \sigma_{x x}^{\mathrm{eq}}$ was obtained by subtracting $\sigma_{x x}^{e q}$ at the conductivity minimum before DNP from the dc magnetoconductivity minima which were measured during the relaxation decay period immediately following DNP. In this procedure the $\sigma_{x x}\left(B_{0}\right)$ traces were recorded over a small region around $\nu=1$ using multiple up and down field sweeps during a period of about $800 \mathrm{~s}$. The Overhauser shift obtained at the termination of the DNP down-sweep was $\delta B_{n}(t=0) / B=0.021$. This value and $T_{1 n}$ $=217 \mathrm{~s}$ were used to plot the expression $\delta \sigma_{x x}(t) / \sigma_{x x}^{\mathrm{eq}}=\alpha\left(\delta B_{n} / B\right) \exp \left(-t / T_{1 n}\right)$ in Fig. $3 \mathrm{~b}$. The value of $\alpha$ is based on the $g$ factor measured by ESR. Quantitative agreement between this model and the experimental data is obtained with no adjustable parameters. 

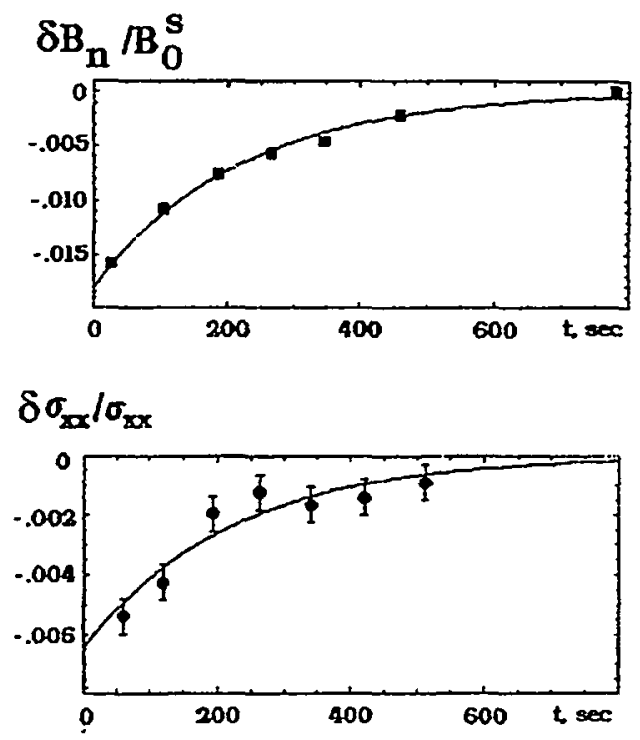

FIG. 3. Time dependence of the relative Overhauser shift $\delta B_{n} / B_{0}^{3}$ of the 2D electron spin resonance following DNP at $T=2.5 \mathrm{~K}$ in sample EA216. The solid line represents an exponential fit to the data. The relaxation time obtained from the fitting is $T_{1 n}=217 \mathrm{~s}$. Part (b) shows the time dependence of the relative variation of the longitudinal conductivity, $\delta \sigma_{x x} / \sigma_{x x}$, obtained by a sequence of magnetic field up-sweeps following an initial DNP down-sweep. The solid line represents a theoretical calculation of the effect using (4) with no adjustable parameters.

In conclusion, we have observed the effect of the nuclear hyperfine field on the dc conductivity of $2 \mathrm{D}$ electrons under quantum Hall effect conditions in AlGaAs/GaAs multiquantum wells at filling factor $\nu=1$. The nuclear spin polarization was enhanced using dynamic nuclear polarization, whereby the nuclei become polarized due to cross relaxation with electrons being driven by ESR transitions into a nonequilibrium polarization state. The hyperpolarized nuclei produce a local hyperfine field which is experienced by the electron spins. The resulting Overhauser shift of the ESR transition was measured using electrically detected ESR. The value of the dc conductivity change due to the nuclear spin polarization corresponds quantitatively to the thermal activation model incorporating the nuclear hyperfine field into the Zeeman energy of the charged groundstate excitations. The dc conductivity decay time measured experimentally is, within experimental error, the same as the nuclear spin-lattice relaxation time observed via Overhauser shift measurements near filling factor $\nu=1$. These findings unequivocally establish the nuclear-spin origins of the observed conductivity changes.

We thank N. Bonesteel, L. Engel, J. Graybeal, I. Kukushkin, N. Sibeldin, D. Thouless, and I. D. Vagner for helpful discussions. We also express our appreciation to Mark Whitton and Bobby Joe Pullum for experimental help, and to Bruce Brandt for superb management of the NHMFL facilities. This work was supported by NSF Grant CHE-9624243 and Project 97-1040, Physics of Solid State Nanostructures, Russia. The work at Sandia was supported by the U. S. Department of Energy under Contract DE- 
AC04-94AL85000. A portion of this work was performed at the NHMFL, which is supported by NSF Cooperative Agreement DMR-9527035 and by the State of Florida.

'C. P. Slichter, Principles of Magnetic Resonance, Springer, Berlin, 1991.

${ }^{2} \mathrm{~J}$. Winter, Magnetic Resonance in Metals, Clarendon Press, Oxford, 1971.

${ }^{3}$ I. D. Vagner and T. Maniv, Physica B 204, 141 (1995).

${ }^{4}$ V. Privman, I. D. Vagner, and G. Kventsel, Phys. Lett. A 239, 141 (1998).

${ }_{5}^{5}$ A. M. Dugaev, I. D. Vagner, and P. Wyder, JETP Lett. 64, 211 (1996).

${ }^{6}$ B. Sapoval, D. Kaplan, and G. Lampel, Solid State Commun. 9, 1591 (1971).

${ }^{7}$ N. Gauss and A. G. M. Jansen, Czech. J. Phys. 46, S4, 2037 (1996).

${ }^{8}$ M. Dobers, K. von KJitzing, J. Schneider, G. Weinmann, and K. PLoog, Phys. Rev. Lett. 72, 1650 (1988).

${ }^{9}$ A. Berg, M. Dober, R. R. Gerhardts, and K. von Klitzing, Phys. Rev. Lett. 64, 2563 (1990).

${ }^{10}$ A. Schmeller, J. P. Eisenstein, L. N. Pfeiffer, and K. W. West, Phys. Rev. Lett. 75, 4290 (1995).

${ }^{11}$ F. F. Fang and P. J. Stiles, Phys. Rev. 174, 823 (1968).

${ }^{12}$ D. Paget, G. Lampel, B. Sapoval, and V. I. Safarov, Phys. Rev. B 15, 5780 (1977).

${ }^{13}$ A. Abragam, The Principles of Nuclear Magnetism, Clarendon Press, Oxford, 1961, p. 191.

${ }^{14}$ A. W. Overhauser, Phys. Rev. 92, 411 (1953).

${ }^{15}$ S. A. Vitkalov, JETP 82, 994 (1996).

${ }^{16} \mathrm{D}$. Stein, K. von Klitzing, and G. Weimann, Phys. Rev. Lett. 51, 130 (1983).

${ }^{17} \mathrm{C}$. R. Bowers, Solid State Nucl. Magn. Reson. 11, 11 (1998), and references therein.

Published in English in the original Russian journal. Edited by Steve Torstveit. 